Objectives To review the safety and efficacy of LAM in reducing the perinatal transmission of HBV.

Methods Medical charts of HBV positive women who received treatment with LAM and who booked for antenatal care between 2007 and 2012 were retrospectively reviewed.

Results Between 2007 - 2012, 34 pregnant HBV positive women received treatment with LAM during the third trimester. All were HbeAg positive, and 6/34 were anti-HbCore IgM positive, indicative of acute infection. Where tested, the predominant genotypes were B and C, occurring in 16/32 and 11/32 respectively. Genotype $\mathrm{D}$ was noted in $4 / 32$ women. One woman was co-infected with Hepatitis C.

Mean viral load (VL) pre-treatment was $>1 \times 108 \mathrm{IU} / \mathrm{ml}$, mean $\mathrm{VL}$ closest to delivery was $6.5 \times 106 \mathrm{IU} / \mathrm{ml}(\mathrm{P}<0.001)$. No resistance to LAM was identified in the $70 \%$ who were tested post treatment.

Median delivery gestation was 39 weeks (range 37-41 weeks); $17 / 33$ had a normal vaginal delivery, $5 / 33$ had an instrumental delivery, 9/33 had a $C$ section, 2 delivered elsewhere and one patient is still pregnant. Median birth weight was $3.49 \mathrm{~kg}$ (range $2.33-4.72 \mathrm{~kg}$ ). All babies received HBV IgG and the first dose of vaccine within the first 24 hours of life. Of 33 live born infants, 17 were not infected, 8 left the country prior to the 8-month serology test, 6 have serology pending (not yet 8 months) and 2 were lost to follow up.

Conclusions Treatment with LAM is a safe and effective. No vertical transmission of HBV was noted, and no adverse maternal or fetal effects were reported.

\section{PM.63 INTERNATIONAL VOLUNTEERING TO PROMOTE MATERNAL AND NEWBORN HEALTH: OPTIMISING IMPACT}

doi:10.1136/archdischild-2013-303966.145

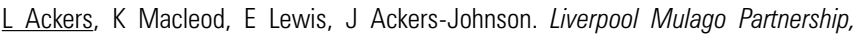
Liverpool, UK

This paper presents the results of an interim evaluation of an initiative funded by the Tropical Health Education Trust (THET) and hosted by the Liverpool-Mulago-Partnership. Internationalisation is becoming an essential dimension of clinical careers resulting in a growth in professional volunteering in developing countries. The Ugandan Maternal and Newborn Hub, formed in 2011 seeks to reduce maternal and newborn mortality in Uganda through improved partnership.

In 2012 the HUB was awarded funding through THET's Health Partnership Scheme to set up the 'Sustainable Volunteering Project' (SVP). The SVP is responsible for the recruitment, deployment and evaluation of professional volunteers across the HUB. It aims to:

- Reduce Maternal and Newborn Mortality in Uganda through the placement of professional volunteers.

- Develop, promote and evaluate a model for sustainable and effective professional voluntarism

Working in close partnership with the Association of Anaesthetists of Great Britain and Ireland, the Royal College of Obstetricians and Gynaecologists, the Royal College of Paediatrics and Child Health and the Royal College of Midwives the SVP aims to provide a more supportive and effective environment for clinical volunteering.

The paper presents results of the interim evaluation outlining key areas of intervention including the reduction of caesareansection rates through instrumental (vacuum) delivery; the promotion of Early Warning Scoring Systems; Infection Control and High Dependency Maternal/Neo-natal Care. It assesses the role of clinical volunteers in promoting sustainable change in development settings and in terms of the acquisition of clinical skills and experience for early career clinicians returning to the UK.

\section{PM.64 PREGNANCY OUTCOMES IN WOMEN WITH SICKLE CELL} DISEASE AT A LONDON HOSPITAL (2006-2012)

doi:10.1136/archdischild-2013-303966.146

J Ip, M Parisaei, E Dorman, K Erskine, T Ahmed, R Amos. Homerton University Hospital NHS Foundation Trust, London, UK

Introduction SCD is associated with both maternal and fetal complications including pre-eclampsia, growth restriction and stillbirth together with an increased frequency of acute painful crises.

Method This was a 6-year retrospective audit of 56 pregnant women with SCD: $22 \mathrm{HbSS} \& \mathrm{HbS}-$ beta(0); $27 \mathrm{HbSC}$; 7 HbS-beta(+).

Setting The women were all managed by the same multidisciplinary team, which included haematolgists, obstetricians and a specialist SCD midwife.

Results There were few obstetric antepartum complications (2\% pre-eclampsia, $2 \%$ antepartum haemorrhage). However, $35 \%$ were admitted with acute painful crises. 7 women underwent regular exchange transfusions for severe pre-existing maternal disease and significant obstetric history. There were no maternal deaths in this cohort.

$9 \%$ of women with SCD were delivered before 34 weeks. $64 \%$ of women were delivered by caesarean section (61\% emergency, majority of which were for failure to progress).

Out of the 56 pregnancies, there were 54 live births, 2 stillbirths (one unexplained at 40 weeks, one with severe growth restriction at 27 weeks), and 1 neonatal death (day 8 secondary to disseminated herpes simplex).

Discussion The Green-top Guideline (No.61, 2011) suggests all those with SCD should be on low dose aspirin from 12 weeks, have appropriate management of painful crisis and undergo extra scanning with uterine artery dopplers.

Most of these pregnancies predate this RCOG guideline. It would be interesting to note if further improvements in outcome will follow recent recommendations. SCD is the commonest and fastest growing single gene genetic disorder in the UK and these women benefit from specialised multidisciplinary care.

\section{PM.65 TO EVALUATE THE EFFECTS OF ANTI-PSYCHOTIC MEDICATIONS TAKEN IN PREGNANCY, COMPARED TO ANTI-DEPRESSANTS AND A CONTROL GROUP WHO HAD MENTAL ILLNESS, BUT WERE NOT ON MEDICATION. THE SETTING WAS A SPECIALIST ANTENATAL LIAISON MENTAL HEALTH CLINIC}

doi:10.1136/archdischild-2013-303966.147

'LE Elbeshir, ' ${ }^{2} \mathrm{SP}$ Pretlove, ${ }^{2} \mathrm{FC}$ Coccia, ${ }^{2} \mathrm{EP}$ Paschetta, ${ }^{1} \mathrm{KI}$ Ismail, ' ${ }^{1} \mathrm{EG}$ Giles. ${ }^{1}$ Birmingham Women's Hospital, Birmingham, UK; ${ }^{2}$ Mother and baby unit at Barberry, Birmigham, UK

Objective Perinatal mental illness presents challenges to obstetricians. Mental illness of all severities can have in impact on maternal and infant outcomes. There is an increasing evidence for the use of anti-depressants in pregnancy, but a paucity of data for women using anti-psychotics.

Design A retrospective casenote review of all cases attending an antenatal mental health clinic between April 2011-12.

Setting Background fetal growth restriction rate 13\%

Results 282 women were referred to the clinic. 215 had depressive disorders, with 139 (76.2\%) on psychotropic medication $132(87.4 \%)$ of the women were on antidepressants and $18(12 \%)$ on anti-psychotics. $28(20 \%)$ babies were below the $10^{\text {th }}$ customised centile \& 19 (13\%) were diagnosed with a congenital abnormality at birth. 72 had anxiety disorders with $32(63 \%)$ on antidepressants and, $10(20 \%)$ on atypical anti-psychotics, 5 (10\%) had babies diagnosed with anomalies at delivery. 14 (29\%) babies in this group were below the $10^{\text {th }} .5$ women with psychotic disorders were all on antidepressants and anti-psychotics. 2 (40\%) of the babies were below 
the $10^{\text {th }}$ centile. Ten women had bipolar disease and 6 used antipsychotics. One $(16 \%)$ baby was below the $10^{\text {th }}$ percentile.

In total 12 women took olanzepine and of these 8 (66\%) babies had a congenital anomaly, 4 had tongue tie but 3 babies (on fluoxetine and olanzepine) had major congenital heart disease.

Conclusion Women referred to a mental health clinic had increased rates of growth restriction compared with the general population. Numbers are small but association of antenatal anti-psychotics with fetal growth restriction and congenital anomalies needs to be considered.

\section{PM.66 WITHDRAWN BY AUTHOR}

\section{PM.67 BOWEL PERFORATION FOLLOWING SEVERE CONSTIPATION IN PREGNANCY: A CASE REPORT}

doi:10.1136/archdischild-2013-303966.148

SJ Bonner, J Ford, F Soydemir. Royal Preston Hospital, Lancashire, North West, UK

Stercoral perforation is defined as "perforation of the bowel due to pressure from a faecal mass." This is due to an accumulation of stool that has hardened and has remained in the bowel over a long period of time causing stagnation and deformity of the large intestine.

At 25 weeks gestation a lady attended with severe constipation despite various stimulants/enemas and left sided abdominal pain. She started vomiting and felt generally unwell. She had abdominal distension and sluggish bowel sounds. Haematology and biochemistry were normal. Ultrasound showed large amounts of gas in the bowel but was otherwise unremarkable. The surgeons reviewed her, excluded any obstruction and continued conservative management with observation and laxatives. Her pain and constipation continued until she became tachycardic and tachyopneic, her CRP began to rise. Prophylactic steroids were given. Antibiotics were commenced and urgent MRI arranged. MRI showed no obvious stricture or structural obstruction. A Naso-gastric tube was passed draining bilious fluid. Throughout the day her clinical condition deteriorated. She was transferred to delivery for critical care review and surgical input.

She eventually went to theatre for a de-functioning iliostomy. At laparotomy bowel perforation was identified with faecal peritonitis. She had a colectomy. Due to maternal condition and access and post operative considerations the baby was delivered by caesarean section.

Although constipation is common amongst pregnant women it is usually corrected by conservative measures such as stimulants/ enemas and adequate hydration. Refractory cases should prompt early referral for investigation for underlying pathology.

\section{PM.68 MANAGEMENT DILEMMAS OF CONN'S SYNDROME IN PREGNANCY}

doi:10.1136/archdischild-2013-303966.149

JE Ford, SJ Bonner, S Gupta. Royal Preston Hospital, Preston, North West, Lancashire, UK

The most common cause of primary hyperaldosteronism is Conn's syndrome $(80 \%)$, and treatment resistant hypertension with hypokalaemia in pregnancy should prompt investigation for this. There is physiological up-regulation of the rennin-angiotensin system during pregnancy, resulting in secondary hyperaldosteronism. The true incidence of Conn's syndrome in pregnancy is unknown but low, and vague presentations make diagnosis and management a dilemma.

A 25 year old primigravida presented at 26 weeks with hypertension, proteinuiria and bilateral leg oedema. She had an emergency caesarean section for severe pre eclampsia. Persistent hypokalaemia lead to a subsequent diagnosis of Conn's syndrome. She had normal adrenal imaging. She began pharmacological treatment with spironolactone. Currently she is 24/40 into her second pregnancy.

Spironolactone is contraindicated due to its teratogenic effects particularly virilisation of male fetuses. She has been commenced on low dose Aspirin and oral potassium supplements. Her renal function and Magnesium levels, along with blood pressure and urinalysis are being monitored regularly.

Studies have shown unsatisfactory hypotensive effects with methyldopa, hydralazine, labetolol, diazoxide and nifedipine. There is limited research available for eplenerone which is structurally similar to spironolactone but weaker and so does not have virilising effects, this is however unlicensed. Although the presentation of pathology can be non specific and "normal" in early pregnancy, treatment resistant hypertension requires assessment for primary hyperaldosteronism. Management has considerable difficulties due to the teratogenicity/lack of evidence on first line pharmacological treatments. So far there is supportive evidence that amiloride is safe and effective and could be used in these cases.

\section{PM.69 HAEMATINIC EVALUATION AND SUPPLEMENTATION IN A NORMAL PREGNANCY}

doi:10.1136/archdischild-2013-303966.150

${ }^{1} \mathrm{CM}$ McCarthy, ${ }^{2} \mathrm{MR}$ Cahill, ' $\mathrm{K}$ O'Donoghue. 'Department of Obstetrics and Gynaecology, Cork University Maternity Hospital, Cork, Ireland; ' 2 Department of Haematology, Cork University Hospital, Cork, Ireland

Maternal and Neonatal complications have been described in antenatal iron, folate and $\mathrm{B} 12$ deficiencies. ${ }^{1}$ International recommendations have described the investigation and treatment of haematinic deficiencies. ${ }^{2}$

A prospective audit was conducted between January and April 2012. Postnatal patient charts were randomly sampled. Data was entered into a secure database. Haematinic levels were retrospectively acquired using institutional laboratory systems.

176 patients were included in our sample group, with a total of 757 investigations. 155 patients had more than 2 sets of haematological investigations during pregnancy. $39.7 \%$ had haematinic investigations performed. 48 patients had a ferritin level below $30 \mathrm{ug} / \mathrm{L}$, of which 11 were using iron supplementation. $69.8 \%$ of patients reported folate supplementation, with $12.5 \%$ taking combined antenatal supplementation. $21 \%$ were taking a form of iron supplementation. Of the 28 people who were recorded as not taking antenatal supplementation, 6 had suboptimal ferritin levels.

Currently, there are no national guidelines on haematinic investigation in the antenatal population. This is imperative to improve patient outcomes. It is also essential to treat those who demonstrate clinical anaemia, and sub-optimal ferritin levels.

\section{REFERENCES}

1. Scholl, Hediger. Anemia and iron-deficiency anaemia: compilation of data on pregnancy outcome. Am J Clin Nutr 1994;59:492S-500S.

2. Pavord, Myers, Robinson, Allard, Strong, Oppenheimer. UK guidelines on the management of iron deficiency in Pregnancy. British Committee for Standards in Haematology. July 2011.

\section{PM.70 COMPARATIVE STUDY BETWEEN GESTATIONAL AND PREGESTATIONAL DIABETES IN RELATION TO GLYCEMIC CONTROL AS REGARDING FETAL AND NEONATAL OUTCOME}

doi:10.1136/archdischild-2013-303966.151

M Bahaa. Kasralainy Maternity Hospital, Cairo, Egypt

Background Pregnancy tends to reset the glucose homeostasis in the direction of diabetes. About $1-2 \%$ of all pregnant women 